\title{
Strain enhanced lithium adsorption and diffusion on silicene
}

\author{
Xiao Wang, ${ }^{a}$ Youhua Luo, ${ }^{a}$ Ting Yan, ${ }^{b}$ Wei Cao ${ }^{c}$ and Meng Zhang ${ }^{{ }^{a}}$
}

${ }^{a}$ School of Science, East China University of Science and Technology, Shanghai 200237, China.

${ }^{\mathrm{b}}$ Wuhan Museum of Science and Technology, Wuhan, 430063, China

c Nano and Molecular Systems Research Unit, P.O. Box 3000, FIN-90014, Finland

* Corresponding author: E-mail: mzhang@ecust.edu.cn 


\begin{abstract}
The performance of Li-ion batteries relies heavily on the capacity and stability of constituent electrodes. Recently synthesized 2D silicene has demonstrated excellent Li-ion capacity with high charging rates. To exploration of the external influences for battery performance, in this work, first-principles calculations are employed to investigate the effect of external strain on the adsorption and diffusion of $\mathrm{Li}$ on monolayer silicene. It is found that tensile strain could enhance Li binding on silicene. The diffusion barrier is also calculated and the results show that Li diffusion through silicene is facilitated by tensile strain, whereas the strain have limited effect on the energy barrier of diffusion parallel to the plane of pristine silicene. Our results suggest that silicene could be a promising electrode material for lithium ion batteries.
\end{abstract}




\section{Introduction}

The increasing applications of lithium ion batteries (LIBs) in portable electronic devices and electric vehicles, have prompted considerable research efforts to develop new electrode material with high energy capacity. ${ }^{1,2}$ Silicon-based materials have recently attracted a lot of attention as promising candidate for anode material of next-generation LIBs, because Silicon (Si) has extremely high theoretical lithium (Li) capacity $(4200 \mathrm{mAh} / \mathrm{g})$, which is about ten times as large as that of graphite $(372 \mathrm{mAh} / \mathrm{g}) .^{3-5}$ However, the high capacity of $\mathrm{Si}$ is generally associated with serious volumetric expansion $(\sim 400 \%)$ upon lithium insertion, which cause a rapid decay of 
Si electrode performance during charging/discharging cycle. ${ }^{6,7}$ In order to improve this problem, a number of methods have been put forward in theory and experiment, such as through $\mathrm{Si}$ structural modification: porous structure, ${ }^{8,9}$ nanowires ${ }^{10-12}$ and nanoparticles, ${ }^{13}$ et al.

Most recently, silicene, a novel graphene-like allotrope of silicon has been successfully synthesized on various metal substrates such as $\mathrm{Ag}$, Ir and $\mathrm{ZrB}_{2}{ }^{14-17}$ Similar to graphene, silicene provides a large surface area for adsorption and migration of Li. Theoretical studies have shown that the binding energy between Li and single-layer silicene is $\sim 2.2 \mathrm{eV}$ per $\mathrm{Li}$ atom while the barriers for $\mathrm{Li}$ diffusion are less than $0.4 \mathrm{eV},{ }^{18,19}$ much lower than that of Li diffusion on graphene. ${ }^{20,21}$ Moreover, in contrast to crystalline silicon, silicene does not suffer from irreversible structural changes during lithiation and delithiation cycles and the associated change in the effective volume is small. ${ }^{18}$ Thus, silicene provides promising candidate materials for the electrodes of Li-ion batteries. Although silicene can overcome the structural failure of active electrode materials to some extent, it undergoes some deformation in the silicene plane. Moreover, strain in nanomaterials has a great impact on the $\mathrm{Li}$ storages due to volume change. However, the characteristics of $\mathrm{Li}$ adsorption and diffusion on silicene under external strain, which are especially important for battery performance, are still not investigated. Thus, in order to evaluate this effect, we pursue theoretical analysis for some insights into the energy barriers and adsorption energy during Li diffusion on silicene.

In this work, we present a series of atomistic simulations to study Li binding and 
diffusion in strained silicene by using first-principles calculations, which is a powerful method to investigate Li diffusion in electrode materials. ${ }^{18-22}$ Two diffusion pathways are considered, Li diffusion parallel to pristine silicene and through a silicene sheet. For the most stable Li adsorption sites, the effect of strain on the Li adsorption energy is also evaluated. In addition, by means of energy barriers, the lithium diffusion kinetics is explored under different strains.

\section{Computational methods}

In our studies, all the calculations are performed within the framework of unrestricted spin-polarized DFT, implemented in the Dmol3 code. ${ }^{23-25}$ The PerdewBurke-Ernzerhof (PBE) functional within the generalized gradient approximation (GGA) is used as the exchange correlation functional. ${ }^{26}$ The all-electron calculations and a double numerical basis set plus polarization functional (DNP) are adopted. ${ }^{24}$ The global orbital cutoff of $4.6 \AA$ are set in the spin-unrestricted calculations for all systems, and the effect of periodic boundaries was negligible. The dispersion corrections of Tkatchenko-Scheffler van der Waals ${ }^{27}$ are also included in the calculation. A $5 \times 5 \times 1$ supercell with periodic boundary condition is used to model the infinite silicene sheet. The vacuum space of $20 \AA$ was set in the direction normal to the sheets to avoid the interactions between periodic images. The Brillouin zone is represented by the set of $8 \times 8 \times 1$ k-points ${ }^{28}$ for the geometry optimizations, and $15 \times$ $15 \times 1 \mathrm{k}$-points are used to obtain the density of states (DOS). Geometric structures of 
the systems studied were fully relaxed by minimizing the forces on each atom and the total energy change to below $0.001 \mathrm{Ha} / \AA$ and $10^{-6} \mathrm{Ha}$, respectively.

To analyze the stability of $\mathrm{Li}$ adsorbed on silicene, the binding energy $\left(\mathrm{E}_{\mathrm{b}}\right)$ of a lithium atom with the pristine and stained silicene substrates are given by

$$
\mathrm{E}_{\mathrm{b}}=\mathrm{E}_{\mathrm{Li}+\text { strained }}-\left(\mathrm{E}_{\text {strained }}+\mathrm{E}_{\mathrm{Li}}\right)
$$

where $\mathrm{E}_{\mathrm{Li}+\text { strained }}$ is the total energy of the adsorption system of the silicene, $\mathrm{E}_{\text {stained }}$ is the energy of silicene, and $\mathrm{E}_{\mathrm{Li}}$ is the energy of an isolated $\mathrm{Li}$ atom.

In searching the diffusion transition state (TS), a linear synchronous transit (LST) is carried out and the conjugated gradient minimizations and quadratic synchronous transit (QST) maximizations ${ }^{29}$ are then repeated until a TS structure has been determined. All determined TS structures are validated and the diffusion paths are found using the nudged-elastic-band method. ${ }^{30}$

\section{Results and discussion}

\subsection{Effect of strain on Li adsorption}

To analyze the adsorption of $\mathrm{Li}$ atom on silicene, we consider four typical sites with high symmetry: the site on the top of a $\mathrm{Si}$ atom in higher plane (T), the site on the top of a Si atom in lower plane (V), the site at the midpoint of a Si-Si bond (B), and the site in the center of a hexagon $(\mathrm{H})$. The corresponding sites are shown in Figure 1. After full relaxation, the $\mathrm{H}$ site of silicene is the most stable adsorption 
position, where Li resides above the center of the hexagonal silicene ring and it has three nearest neighbor Si atoms. The corresponding binding energy is $-2.41 \mathrm{eV}$, which is agreed with other previous works. ${ }^{18,19,22}$ The second favorable adsorption site is the valley (V) site on top of the lower silicon atoms with the binding energies of $-2.02 \mathrm{eV}$. In this configuration, the silicon atom directly below the adatom undergoes a great displacement towards the outside of the plane due to the electrostatic repulsion, as shown in Figure 2(b). Consequently, the Li adatom also prefers to interact with the neighboring three $\mathrm{Si}$ atoms when it is adsorbed on the $\mathrm{V}$ site of the silicene. The adsorption of $\mathrm{Li}$ on the $\mathrm{T}$ site of silicene with a binding energy of $-1.83 \mathrm{eV}$ has a similar structure of that on the V site. Though the valley and top site adsorptions are possible, bridge site adsorption of $\mathrm{Li}$ is not possible on a silicene lattice in our calculation, which is agreed with the results of Sahin et al. ${ }^{31}$

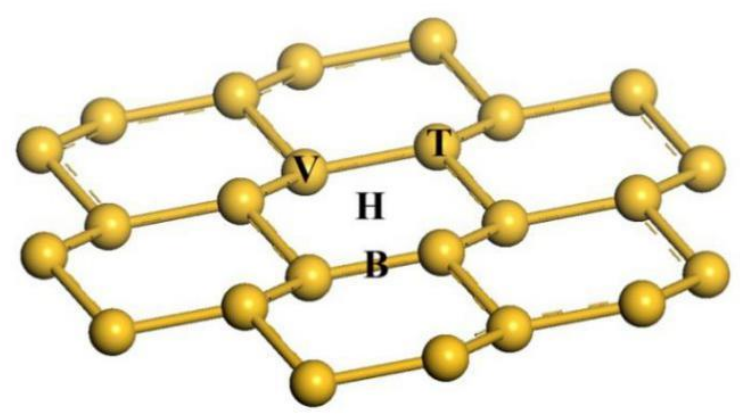

Figure 1. Different adsorption sites of lithium atom on silicene. 
(a)

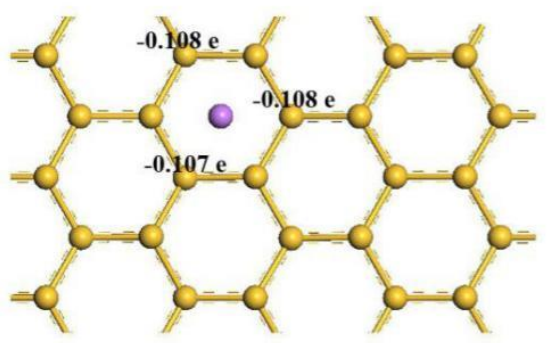

$0.243 \mathrm{e}$

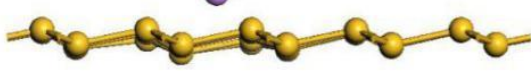

(b)
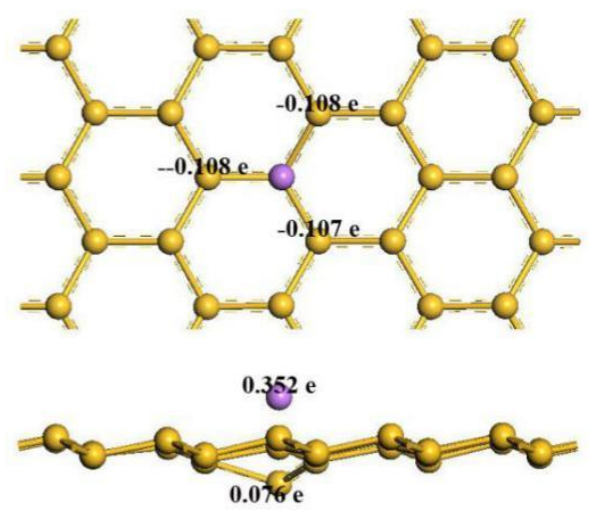

Figure 2. The stable configurations of Li adsorption on (a) $\mathrm{H}$ site and (b) $\mathrm{V}$ site of silicene after optimization. Charge of atoms nearest the adatom calculated by Mulliken population are labelled in black.

In addition, delithiation process was also examined by computing minimum energy path (MEP) of moving Li from the most stable adsorption site to a site $4.0 \AA$ far away above the original one, the results are shown in Figure 3. During the delithiation, the Si atoms near the adatom are first pulled out of the plane, then after removal of the Li atom from the system, the structure of silicene returns to its initial form. Assuming no additional energy barriers to the kinetic transport of $\mathrm{Li}$ in the silicene, lithiation/delithiation should be spontaneous if a potential difference of $\sim 2.4$ $\mathrm{eV}$ is applied. 


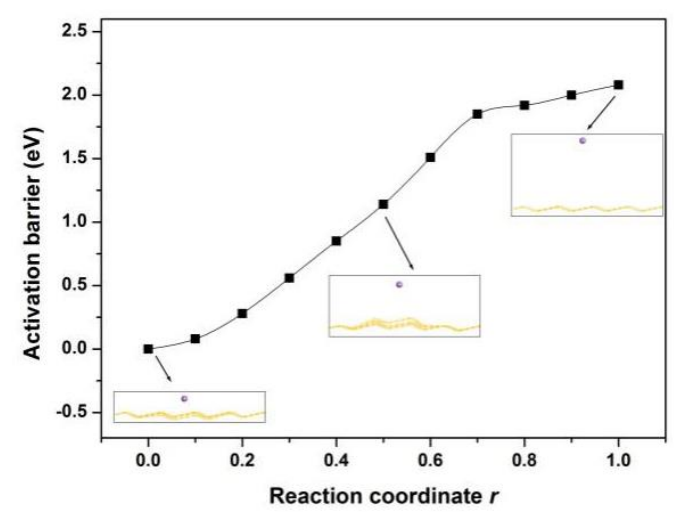

Figure 3. Energy change during delithiation process of the unstrained silicene. The activation barrier is virtually equal to lithium adsorption energy. The variation of activation barrier is plotted along the moving coordinate $r$, which is the migration length normalized by the lattice constant of silicene (3.866 ̊).

For practical application of electrode material for LIBs, the effect of strain on the binding of $\mathrm{Li}$ atom on silicene is examined. The in-plane biaxial tensile strains are imposed on silicene, achieved by enlarging the lattice constants. The strain is set from 0 to $10 \%$ every $2 \%$ was applied to the silicene model, which is within the affordable range of materials strength of silicene. ${ }^{32,33}$ Here the strain is defined as $\varepsilon=\left(a-a_{0}\right) / a_{0}=$ $\Delta \mathrm{a} / \mathrm{a}_{0}$, where $\mathrm{a}_{0}$ and $\mathrm{a}$ are the lattice parameters of the unstrained and strained supercell, respectively. As strain increasing, it is found that all Li atoms can stably adsorb on the $\mathrm{H}$ site of silicene. Specifically, it is noted that when the strain is upon $6 \%$, lithium atom at the $\mathrm{V}$ site or $\mathrm{T}$ site cannot be stably adsorbed and would automatically transfer to the $\mathrm{H}$ site. Taking $\mathrm{Li}$ adsorption at the $\mathrm{V}$ site as an example, when tensile strain increases, more charge transfer from $\mathrm{Li}$ atom to silicene. 
Meanwhile, the buckling of the sheet decreases, which results in the distance between the $\mathrm{Li}$ and the directly interacted valley $\mathrm{Si}$ atom remained. Thus the electrostatic repulsion between $\mathrm{Li}$ and the valley $\mathrm{Si}$ atom increases. On the other hand, the electrostatic attraction between $\mathrm{Li}$ and the neighboring three $\mathrm{Si}$ atoms increase because of the more charge transfer. It is noted that the electrostatic attraction of the lithium atom to the three silicon atoms is not completely symmetrical, and this asymmetry increases with increasing tensile strain. One silicon atom is more attractive to the lithium atom than the other two atoms. Thus the combined increasement of the repulsive force and asymmetric attraction make Li moving to the $\mathrm{H}$ site of silicene when the strain further increases to $6 \%$. The results of binding energies with $\mathrm{Li}$ adsorption on sites of silicene and the related adsorption distance as a function of strain are demonstrated in Figure 4(a). It can be observed that the binding energy of Li is reduced when the strain is applied, which indicates that the interactions between $\mathrm{Li}$ atom and silicene are much sensitive to external strains and implies that tensile strain can enhance the Li binding kinetics. Although the binding energies of Li adsorption on $\mathrm{H}$ or $\mathrm{V}(\mathrm{T})$ sites of silicene are all decreased with increasing strain, the adsorption distance exhibits different characteristics (Figure 4(b)). The adsorption distance of Li at $\mathrm{H}$ site decreases with rising strain, consistent with stronger binding at large strains. Here the adsorption distance is defined as the distance of the adsorbed Li atom to the lowest basal plane of silicene sheet. Nevertheless, the adsorption distance on the $\mathrm{V}(\mathrm{T})$ site is found to vary little with the increase in external strains among strain changing from $0 \%$ to $4 \%$. This fact is due to the in-plane relaxation of silicene monolayer 
induced by external strains, which results in a deformation in the perpendicular directions. Since silicene sheet is low-buckled, the strain leads to a rise of the bond length between $\mathrm{Si}-\mathrm{Si}$ and makes the silicene more flat. The bond strength of $\mathrm{Si}-\mathrm{Si}$ in silicene also weakens under strain. Therefore, as the strain increases, the binding distances of $\mathrm{Li}$ to the sheet surface are reduced due to their stronger interactions between $\mathrm{Li}$ atom and hexagon ring of silicene. While for Li adsorption on $\mathrm{V}$ site, their binding distances vary little attributes to the strong interaction between $\mathrm{Li}$ and valley $\mathrm{Si}$ atom whether there is strain applied.
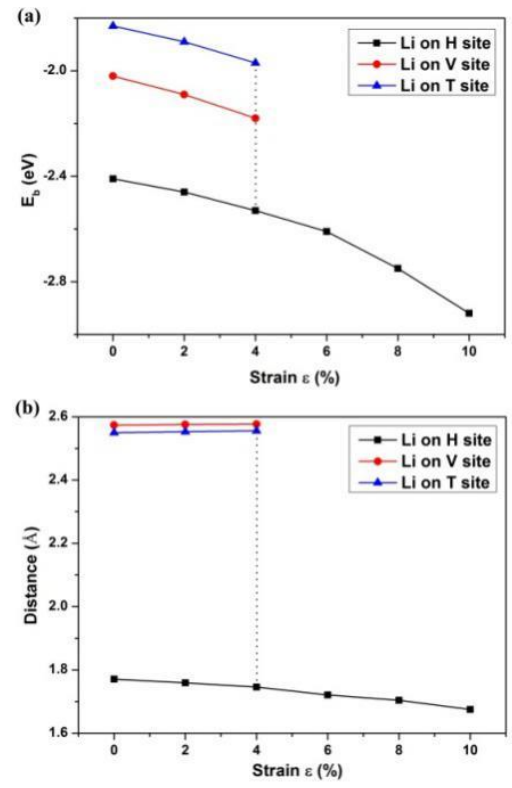

Figure 4. The binding energies (a) and adsorption distance (b) with Li adsorption of silicene as a function of strain. Here, for $\mathrm{Li}$ adsorption on $\mathrm{H}$ site, the adsorption distance is defined as the distance of the adsorbed Li atom to the lowest basal plane of silicene sheet; for Li adsorption on $\mathrm{V}$ or $\mathrm{T}$ site, the adsorption distance is the distance between $\mathrm{Li}$ atom and the adsorbed $\mathrm{Si}$ atom. 
To gain more insight into the adsorption process, a charge analysis is also performed. Li is known as n-type dopant as other alkali metal adatoms. Mulliken charge analysis indicates that $\mathrm{Li}$ atom donates a charge of 0.243 e to the silicene monolayer at strain-free state when it is adsorbed at the $\mathrm{H}$ site. Under external strain, the charge transfer demonstrated in Figure 5 increased monotonically with increasing strains. As such, the Li-Si interactions are energetically favorable to form some ionic-like bond, which accounts for the continuously increase of binding strength by strong electrostatic attractions.

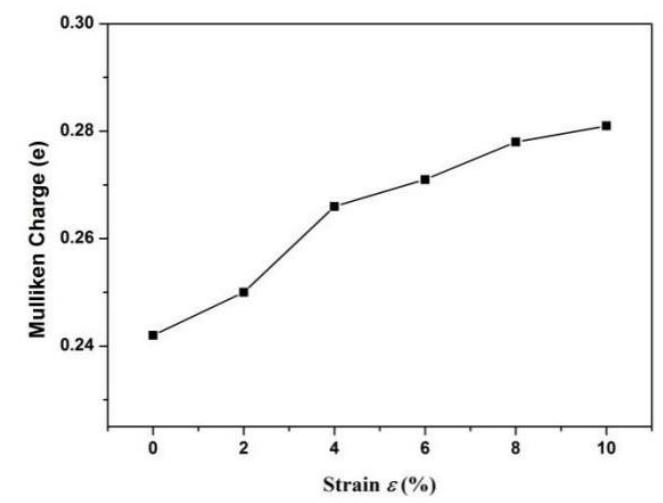

Figure 5. Mulliken charge transfer from Li atom to the silicene sheet under different strain when $\mathrm{Li}$ adsorption on the $\mathrm{H}$ site of silicene.

\subsection{Effect of strain on Li diffusion}

The performance of silicene used in LIBs relies heavily on the diffusion properties of $\mathrm{Li}$ atom. It has been reported that the pristine silicene have a rather low diffusion barrier for Li atoms. We have re-calculated three diffusion paths connecting 
$\mathrm{Li}$ in neighboring $\mathrm{H}$ sites on unstrained silicene. Paths I and II pass on top of Si atoms in the lower and upper sublattice, respectively, while path III directly connects the initial and final $\mathrm{H}$ sites. Path $\mathrm{I}$ is found to result in the lowest diffusion barrier of 0.27 $\mathrm{eV}$ as shown in Figure 6, which is lower than the diffusion barrier of $0.327 \mathrm{eV}$ for a lithium atom migrating on pristine graphene. ${ }^{20,21}$. Considering the difference in lithium concentration, our calculated diffusion barrier agrees well with the theoretical result obtained by Tritsaris et al. ${ }^{18}$ To evaluate the effect of the strain on the diffusion of $\mathrm{Li}$ atom on silicene, we also performed the LST/QST calculations at the same sites obtaining the optimized $\mathrm{Li}$ atom migration pathways and migration energy barriers. The variation of the Li atom migration energy barriers with strain are summarized in Table 1 to reveal the change rule of strain effect on the Li atom migration process. It can be observed that the energy barrier of Li migration is affected very little by strain which slowly declines on the strains $\varepsilon$ range. Still, a smaller driving force should be needed to facilitate Li migration parallel to the silicene sheet. In contrast to the little effect on the energy barrier, we find that the pathways have significantly changed by comparing the $\mathrm{Li}$ atom migration path under strain $4 \%$ before and after. We respectively given the optimized migration pathway and energy profile with strain $4 \%$ and $6 \%$ in Figure 7. It is found that when the strain is small, $\mathrm{Li}$ atom diffusion pathway follows the way it migrates parallel the pristine siliene $(\mathrm{H} \rightarrow \mathrm{V} \rightarrow \mathrm{H})$. After the strain continually increases, $\mathrm{Li}$ atom directly moves from one stable $\mathrm{H}$ site to the nearest $\mathrm{H}$ site with the saddle point bridge site which is similar to the pathway of $\mathrm{Li}$ migration on graphene. ${ }^{20}$ This is due to the strain makes the original adsorption 
configurations change, thus the following migration channel alters. As strain increases, $\mathrm{Li}$ atom at the top of the $\mathrm{Si}$ atom would automatically move to the nearest center of hexagon in silicene and the degree of buckling of silicene becomes lower. The migration process will be as far as possible to select the path of the lower barrier. Thus, the migration path of Li stom will change with the strain.

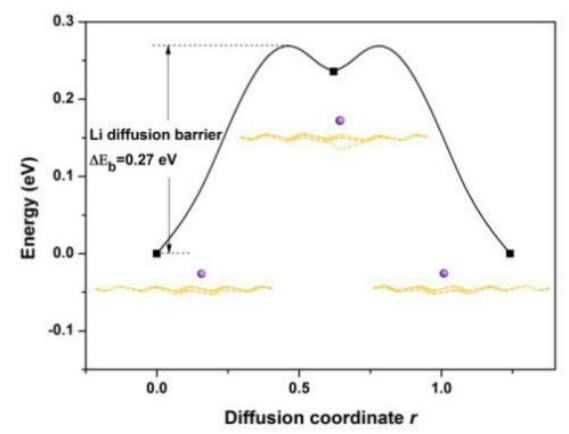

Figure 6. The diffusion path and relative energy profile of Li diffusion parallel to the unstrained silicene. The variation of energy is plotted along the diffusion coordinate $r$, which is the pathway length normalized by the lattice constant of silicene ( $3.866 \AA$ ).

Table 1 . The variation of the Li ion migration energy barriers with strain.

\begin{tabular}{ccc}
\hline Stain $(\varepsilon)$ & $\begin{array}{c}\text { Energy barrier of Li migration } \\
\text { parallel silicene }(\mathrm{eV})\end{array}$ & $\begin{array}{c}\text { Energy barrier of Li moving } \\
\text { through silicene }(\mathrm{eV})\end{array}$ \\
\hline $0 \%$ & 0.27 & 1.37 \\
\hline $2 \%$ & 0.26 & 1.20 \\
\hline
\end{tabular}




\begin{tabular}{lll}
\hline $4 \%$ & 0.25 & 1.05 \\
\hline $6 \%$ & 0.20 & 0.89 \\
\hline $8 \%$ & 0.19 & 0.73 \\
\hline $10 \%$ & 0.18 & 0.59 \\
\hline
\end{tabular}
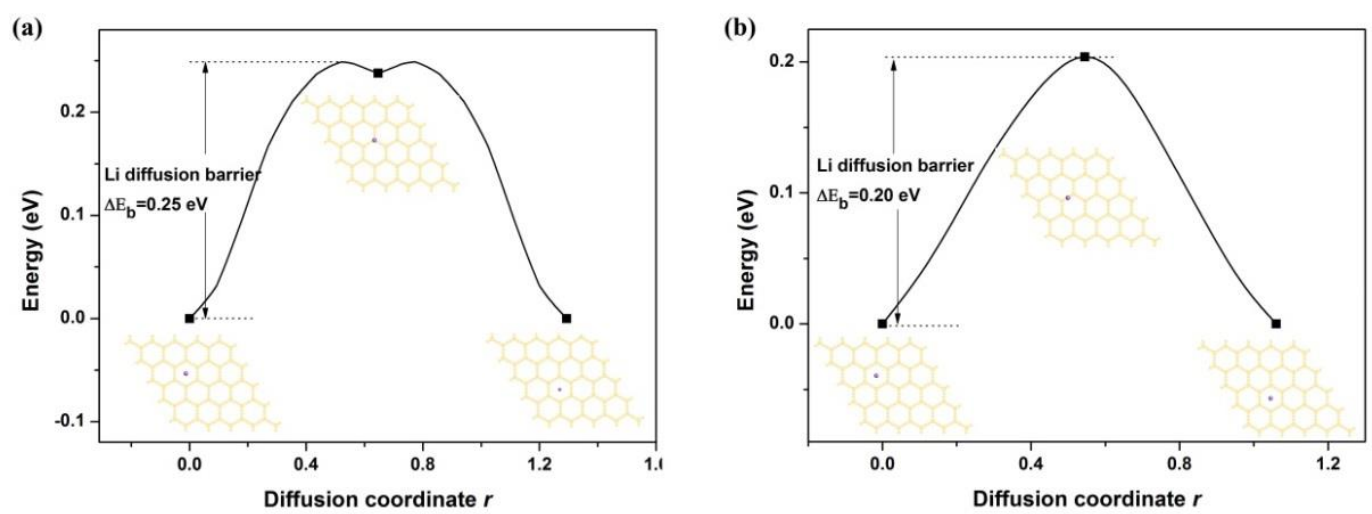

Figure 7. The diffusion path and relative energy profile of Li diffusion parallel to the silicene under strain $4 \%$ (a) and $6 \%$ (b). The variation of energy is plotted along the diffusion coordinate $r$.

The effect of strain on Li diffusion through silicene is also explored. In fact, a lithium atom moves along a straight pathway from one side of silicene to the other side is much easier than that diffusion through a graphene sheet, however, it still need a barrier of $1.37 \mathrm{eV}$ (Figure 8). It can be observed in Table 1 that the energy barrier is greatly reduced with increasing tensile strain during $\mathrm{Li}$ diffusion through the hexagon ring. The energy barrier dramatically reduces to $0.59 \mathrm{eV}$ under a strain of $10 \%$, much 
lower than that for Li diffusion through the pristine silicene, which could greatly increases the diffusion coefficient. This contributes to the extension of hexagon and the weaker bonding between $\mathrm{Si}-\mathrm{Si}$. It is expected to improve the rate capability of lithium-ion batteries.

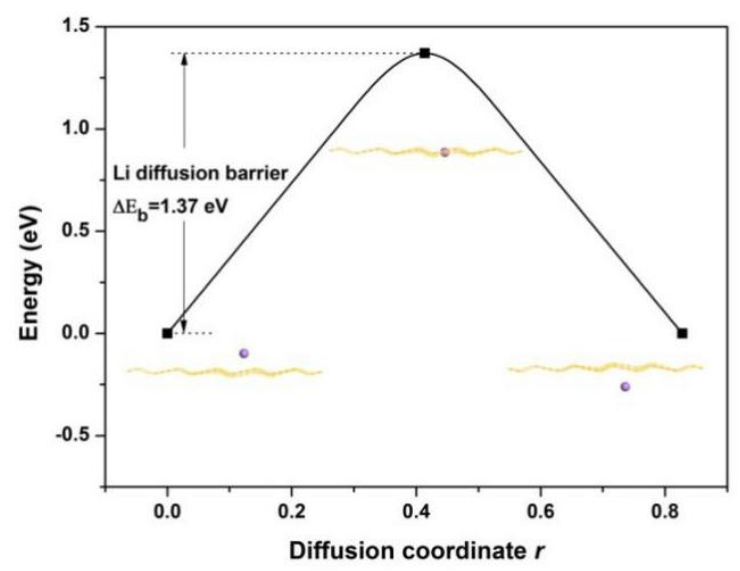

Figure 8. The diffusion path and relative energy profile of Li diffusion through unstrained silicene. The variation of energy is plotted along the diffusion coordinate $r$.

\subsection{Effect of strain on electronic properties of Li adsorbed silicene (Li-silicene)}

The electronic conductivity of electrode materials is also an important essential character. A high rate electrode material of LIBs needs to possess good conductivity. To further understand the strain effect on conductivity of Li-silicene, we summarized their electronic properties with and without strain in Table 2. As previous studies, a semimetal-metal transition occurs when an in-plane strain larger than $7.5 \%$ is applied in silicene. ${ }^{33,34}$ In our work, a similar result has been observed, that when the external 
strain imposed on silicene increases to $8 \%$, its semimetal character changes to metal character. The projected density of states (PDOS) of unstrained Li-silicene is first calculated and shown in Figure 9. It is found that after $\mathrm{Li}$ atom adsorption, semimetallic silicene becomes metallic due to the donation of $\sim 0.243$ e charge from $\mathrm{Li}$ into the silicene conduction band. Here, in accordance to the strong interaction, the Fermi level $\left(\mathrm{E}_{\mathrm{F}}\right)$ is shifted by $\sim 0.2 \mathrm{eV}$. The peak appeared in the vicinity of $0.4 \mathrm{eV}$ above $\mathrm{E}_{\mathrm{F}}$ is attributed to $\mathrm{Li}$ atom adsorption bands formed by the hybridization of adsorbate-s states with the silicene-p states. From Figure 10, when $2 \%$ or $10 \%$ tensile strain is applied, the metallic property of Li-silicene is not influenced. However, the conduction band minimum at $\Gamma$ point $(\mathrm{CBM}-\Gamma)$ with respect to the Fermi level decreases as increasing the tensile strain due to the elongation of the bond lengths. ${ }^{35}$

Table 2. Band gaps of silicene and $\mathrm{Li}$ adsorbed silicene with and without strain.

\begin{tabular}{ccc}
\hline Stain $(\varepsilon)$ & Silicene & Li adsorbed silicene \\
\hline $0 \%$ & Semimetal & Metal \\
\hline $2 \%$ & Semimetal & Metal \\
\hline $4 \%$ & Semimetal & Metal \\
\hline $6 \%$ & Semimetal & Metal \\
\hline $8 \%$ & Metal & Metal \\
\hline
\end{tabular}




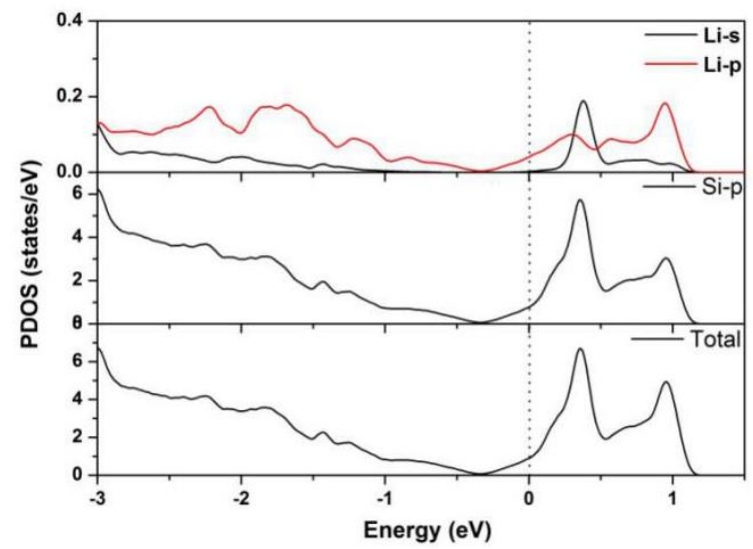

Figure 9. DOS of Li-silicene including $\mathrm{Li}$ and nearest silicon atoms at strain-free states. The PDOS of Li and Si atom are also provided, respectively. Fermi level is set to zero.
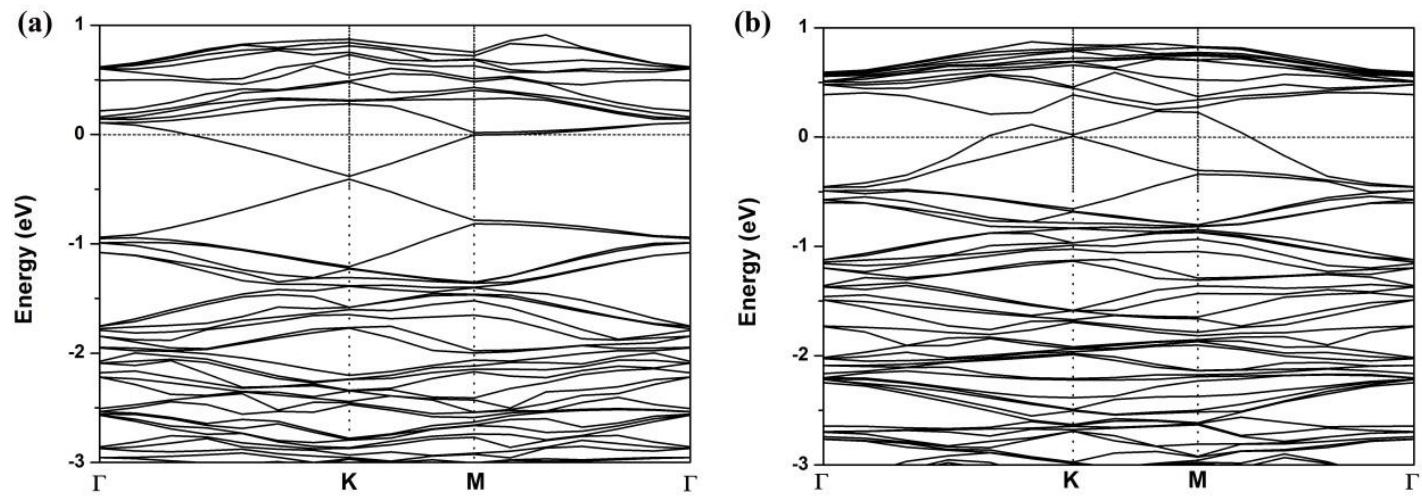

Figure 10. Band structures of Li-silicene under strain of (a) $2 \%$ and (b) $10 \%$. Fermi level is set to zero.

\section{Conclusions}

For silicene modified electrode materials in lithium-ion batteries, silicene would 
experience considerable tensile strain because of the expansion of the electrode during $\mathrm{Li}$ intercalation. Therefore, here we have revealed the role of strain in the $\mathrm{Li}$ adsorption and diffusion of silicene from first-principles calculations. Our results show that biaxial strain facilitates Li adsorption at hollow sites due to increased charge transfer between $\mathrm{Li}$ and silicene which indicates that the $\mathrm{Li}$ storage and capacity of silicene could be increased under even high strain. Besides, the diffusion of $\mathrm{Li}$ on silicene becomes easier by strain, especially for Li diffusion through the sheet. Our results indicate that silicene has great potential as electrode materials used in LIBs with high stability and fast cycling rate.

\section{Acknowledgements}

The authors gratefully acknowledge financial support from NSFC (No. 21303054), and the Fundamental Research Funds for the Central Universities under projects No. 222201414040. All the computation simulation was undertaken with the resources provided from the High Performance Computing Center of East China University of Science and Technology.

\section{Reference}

1 U. Kasavajjula, C. Wang and A. Appleby, J. Power Sources, 2007, 163, 1003.

2 M. Armand and J.-M. Tarascon, Nature, 2008, 451, 652. 
3 B. Boukamp, G. C. Lesh and R. A. Huggins, J. Electrochem. Soc., 1981, 128, 725.

4 C. K. Chan, H. Peng, G. Liu, K. MCilwrath, X. F. Zhang, R. A. Huggins and Y. Cui, Nat. Nanotechnol., 2008, 3, 31.

5 K. Zhao, G. A. Tritsaris, M. Pharr, W. L. Wang, O. Okeke, Z. Suo, J. J. Vlassak and E. Kaxiras, Nano Lett., 2012, 12, 4397.

6 U. Kasavajjula, C. Wang, and A. J. Appleby, J. Power Sources, 2007, 163, 1003.

7 H. Wu and Y. Cui, Nano Today, 2012, 7, 414.

8 M.-H. Park, M. G. Kim, J. Joo, K. Kim, J. Kim, S. Ahn, Y. Cui and J. Cho, Nano Lett., 2009, 9, 3844.

9 T. Song, J. Xia, J. Lee, D. Lee, M. Kwon, J. Choi, J. Wu, S. Doo, H. Chang, W. Park, et al., Nano Lett., 2010, 10, 1710.

10 C. K. Chan, R. N. Patel, M. J. O'Connell, B. A. Korgel and Y. Cui, ACS Nano, $2010,4,1443$.

11 L. Cui, R. Ruffo, C. K. Chan, H. Peng and Y. Cui, Nano Lett., 2009, 9, 491.

12 C. K. Chan, H. Peng, G. Liu, K. McIlwrath, X. F. Zhang, R. A. Huggins and Y. Cui, Nat. Nanotechnol., 2008, 3, 31.

13 X. H. Liu, L. Zhong, S. Huang, S. X. Mao, T. Zhu and J. Y. Huang, ACS Nano, 2012, 6, 1522.

14 B. Feng, Z. Ding, S. Meng, Y. Yao, X. He, P. Cheng, L. Chen and K. Wu, Nano 
Lett., 2012, 12, 3507.

15 P. Vogt, P. De Padova, C. Quaresima, J. Avila, E. Frantzeskakis, M. C. Asensio, A. Resta, B. Ealet and G. Le Lay, Phys. Rev. Lett., 2012, 108, 155501.

16 L. Meng, Y. Wang, L. Zhang, S. Du, R. Wu, L. Li, Y. Zhang, G. Li, H. Zhou, W. A. Hofer and H.-J. Gao, Nano Lett., 2013, 13, 685.

17 A. Fleurence, R. Friedlein, T. Ozaki, H. Kawai, Y. Wang and Y. Yamada-Takamura, Phys. Rev. Lett., 2012, 108, 245501.

18 G. A. Tritsaris, E. Kaxiras, S. Meng, and E. Wang, Nano Lett., 2013, 13, 2258.

19 S. M. Seyed-Talebi, I. Kazeminezhada and J. Beheshtian, Phys. Chem. Chem. Phys., 2015, 17, 29689.

20 X. F. Fan, W. T. Zheng and J. L. Kuo, ACS Appl. Mater. Interfaces, 2012, 4, 2432.

21 C. Uthaisar and V. Barone, Nano Lett., 2010, 10, 2838.

22 J. K. Deng, J. Z. Liu and N. V. Medhekar, RSC Adv., 2013, 3, 20338.

23 B. Delley, J. Chem. Phys., 1990, 92, 508.

24 B. Delley, J. Chem. Phys., 1991, 94, 7245.

25 B. Delley, Int. J. Quantum Chem., 1998, 69, 423.

26 J. P. Perdew, K. Burke and M. Ernzerhof, Phys. Rev. B: Condens. Matter Mater. Phys., 1996, 77, 3865.

27 A. Tkatchenko and M. Scheffler, Phys. Rev. Lett., 2009, 102, 073005. 
28 H. J. Monkhorst and J. D. Pack, Phys. Rev. B: Condens. Matter Mater. Phys., 1976, 13, 5188 .

29 T. A. Halgren and W. N. Lipscomb, Chem. Phys. Lett., 1977, 49, 225.

30 G. Henkelman and H. Jonsson, J. Chem. Phys., 2000, 113, 9978.

31 H. Sahin and F. M. Peeters, Phys. Rev. B, 2013, 87, 085423.

32 Q. Peng, X. D. Wen and S. De, RSC Adv., 2013, 3, 13772.

33 H. J. Zhao, Phys. Lett. A, 2012, 376, 3546.

34 R. Qin, C. H. Wang, W. J. Zhu and Y. L. Zhang, AIP Adv., 2012, 2, 022159.

35 G. Liu, M. S. Wu, C. Y. Ouyang and B. Xu, EPL, 2012, 99, 17010. 